\title{
RECOLORED IMAGE DETECTION VIA A DEEP DISCRIMINATIVE MODEL
}

\author{
Mubeena Afreen, Akhila Badri, Nulu Hari Prasad, Dr. Chukka Santhaiah \\ Department of CSE \\ Institute of Aeronautical Engineering \\ Hyderabad, Telagana, India
}

\begin{abstract}
Image recoloring is a method that can move picture shading or topic and result in an impalpable change in human eyes. In spite of the fact that picture recoloring is one of the most significant picture control procedures, there is no extraordinary technique intended for identifying this sort of imitation. Right now, propose a trainable start to finish framework for recognizing recolored pictures from normal pictures. The proposed arrange takes the first picture and two inferred inputs dependent on light consistency and between channel relationship of the first contribution to thought and yields the likelihood that it is recolored. Our calculation embraces a CNN-based profound design, which comprises of three component extraction squares and an element combination module. To prepare the profound neural system, we blend a dataset contained recolored pictures and relating ground truth utilizing diverse recoloring strategies.
\end{abstract}

Keywords: Illuminant map, Concatenation, Fusion, Recoloring

\section{INTRODUCTION}

Today, a considerable number of photographs are made by various contraptions and scattered by papers, TVs, and destinations in step by step life. Such countless authentic, regulatory and intelligent affiliations use propelled pictures as check of unequivocal events to choose essential decisions, improvement of less cost and compelling image modifying programming, its anything but difficult for performing the image alterations and the distinctive evidence of fabricated images is a great deal of complex through human eye to eye connection. This prompts trouble in the enduring nature of modernized pictures/photographs as real events. In like way, image legitimate strategies for delivered pictures recognizable proof are basic.

Here we acknowledge central purposes of two surfaces similarly as the primary data picture to isolate whether an image is recolored or not. Earlier formed picture disclosure approaches revolve around quantifiable associations of appearance incorporates in the first and adjusted image. For example, the proposed methodology in won't recognize changed pictures if the pixel regard histogram consequent to changing keeps smooth. Our inspiration is to set up a significant discriminative framework for concealing move ID. Moreover, we analyze the most noteworthy estimations including manufacture disclosure methods, concealing move moves close.

\section{A. Forgery Detection Methods}

Fabrication recognition techniques expect to check the validness of pictures and can be comprehensively grouped into two classes: dynamic validation and a loof confirmation. In dynamic verification systems, information concealing procedures are utilized where a few codes are inserted into the pictures during age. These codes are utilized for additional confirming to validate the inventiveness of picture. Dynamic confirmation strategies can be additionally arranged into two kinds: advanced marks and computerized watermarking.

Watermarking installs watermarks into pictures at the hour of picture securing while computerized marks insert some auxiliary data separated from pictures obtaining end to the pictures.

To develop an auxiliary computerized signature utilizing picture content data in the wavelet change space for picture validation. The fundamental disadvantage of these methodologies remains that they should be embedded at the hour of recording, which restricts these ways to deal with uncommonly prepared advanced cameras. Also, the earlier data is fundamental for a confirmation procedure. Uninvolved confirmation likewise called picture legal sciences which has no necessity for earlier data. Computerized picture crime scene investigation depend on the presumption that altering is probably going to modify the fundamental measurements and recognize validness of a picture by distinguishing these irregularities. Most calculations first partition the info picture into different covering squares of various shape and afterward the component extraction from each square happens. At that point, the arranging is done dependent on the highlights. Ultimately, some morphological activities are applied to distinguish the produced locale.

Different systems have been utilized to identify fraud. Latent strategies can be additionally delegated imitation ward techniques and fraud free techniques. Imitation autonomous strategies distinguish fabrications free of falsification type or can manage different sorts of phonies. 


\section{International Journal of Engineering Applied Sciences and Technology, 2020 \\ Vol. 5, Issue 1, ISSN No. 2455-2143, Pages 462-466 \\ Published Online May 2020 in IJEAST (http://www.ijeast.com)}

\section{B. Color Transfer Approaches}

Ongoing developments in computerized picture handling the upgrade of systems made fresh and helpful applications conceivable. Shading control, challenges the unwavering quality of computerized pictures by producing top notch composite recolored pictures. One ordinarily utilized sort of techniques for moving the shading is model put together recoloring based with respect to the measurements of the shading dispersion in pictures.

Reinhard et al proposed a shading move technique by comprehensively moving hues. They apply a straightforward factual examination to forcing one picture's shading qualities on another in the Lab shading space. The shading moving can successfully and proficiently create a persuading yield.

A N-dimensional likelihood thickness capacity and utilize a postprocessing calculation to keep up the slope field of the first picture.

Palette-based recoloring techniques have been proposed starting late. A probabilistic factor diagram model is made to pick up capability with the properties of model models for concealing 2D plans. Evacuate a concealing palette of an image by packing and make a supportive device for recoloring by adjusting a concealing palette. Notwithstanding the way that these recoloring figurings may leave no visual snippets of data, these procedures modify concealed picture surfaces. At this moment, take central purposes of two surfaces to perceive whether an image is recolored.

\section{LITERATURE SURVEY}

R. Chamlawi, A. Khan, and I. Usman et al. expalined about the impact of the convolutional neural framework significance on precision in the tremendous extension affirmation setting. Our noteworthy point is an evaluation of frameworks of improving the significance using a plan with the occasion (3x3) convolution channels, which has shown monster upgrade for the arrangement can be made by sending the significance to $16-19$ weight layers. Here we will similarly exhibits our depictions to various datasets, as where they can get results. At the present time our best performing Convolutional mastermind structures openly expected to empower can investigate on the significant visual portrayals.[12]

G. S. Spagnolo and M. D. Santis et al. proposed another information driven nonexclusive way to deal with oversee performing control parameter estimation. Our proposed framework can be adjusted to work on two or three exceptional controls without requiring a real expert to turn out noteworthy improvements to the proposed strategy. To achieve, we reconstruct the assessment as a request issue by allotting the parameter space into disjoint subsets Therefore, we structure an obliged $\mathrm{CNN}$-based classifier that can remove gathering highlights direct from information likewise as looking over the control parameter respect in a subject picture.
Through a lot of assessments, we exhibited the plausibility of our method utilizing four stand-out sorts of controls.[13]

L. Rosales-Roldan, M. Cedillo-Hernandez, M. NakanoMiyatake, H. Perez-Meana, and B. Kurkoski et al. expalined that at the present time, have organized an enormous, convolutional neural system to update the 1.2 million pictures in the ImageNet LSVRC-2010 challenges into 1000 momentous classes. On the test information, we accomplished top- 1 and top- 5 mess up paces of $37.5 \%$ and $17.0 \%$ which is broadly superior to the past top level. To diminish the overfitting between the layers a procedure called "dropout" is convincing.[14]

\section{PROPOSED TECHNIQUE}

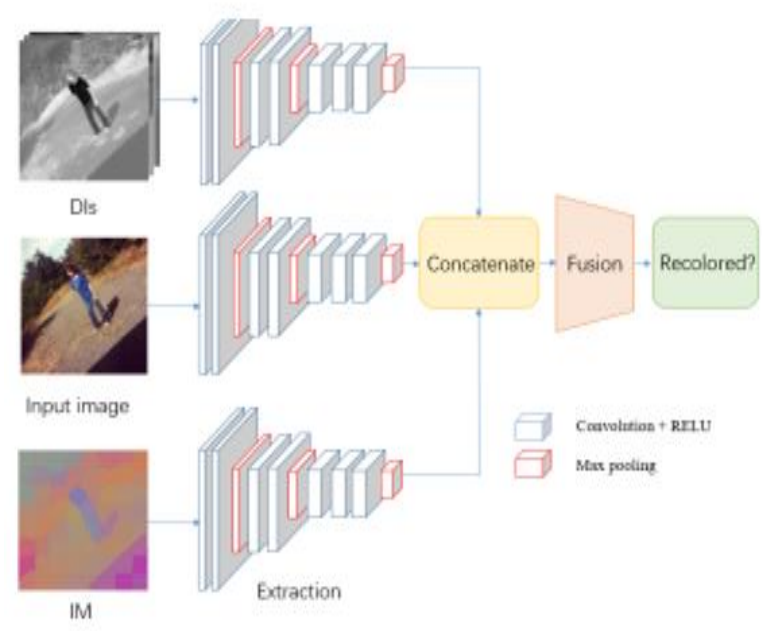

Fig 3.1 Architecture

\subsection{IMAGE FEATURE EXTRACTION}

Here, we eliminate the pictures from dataset, the picture peruser work peruses a lot of training set pictures from dataset, after each picture appointed to compose RGB channel process. we remove the highlights of each information utilizing the initial three convolutional phases of the VGGnet. This stage is equivalent to depiction strategies in conventional techniques.[15]

\subsection{INNER CHANNEL CORRELATION}

This module appraises the relationship of info picture as per the RGB channel, it will be valuable to use this between channel connection for our discriminative assignment. Right now, dimensional consistency, we compute the DIs of R-G, $\mathrm{B}-\mathrm{G}$, and R-B for a picture and place them in the channel measurement as one information part of our system.[16] 
International Journal of Engineering Applied Sciences and Technology, 2020

Vol. 5, Issue 1, ISSN No. 2455-2143, Pages 462-466

Published Online May 2020 in IJEAST (http://www.ijeast.com)

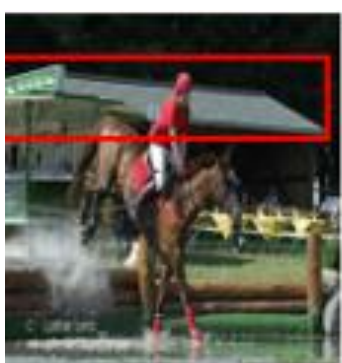

(a) Original image

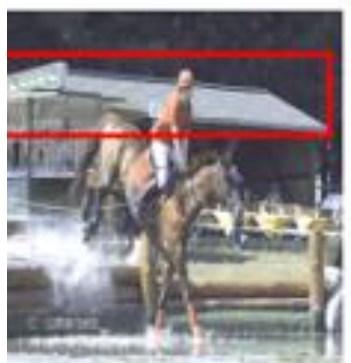

(c) Recolored image

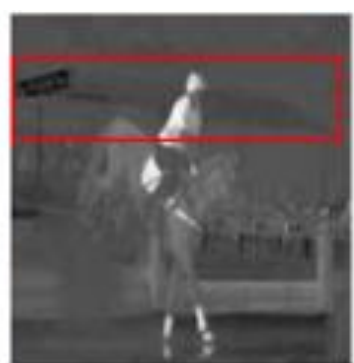

(b) $R-G$ DI of (a)

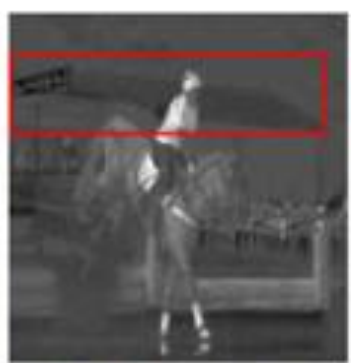

(d) $R-G \mathrm{DI}$ of (c)
Fig 3.2 Comparision of R-G DI's

\subsection{ILLUMINANT IMAGE AND FUSION}

IM speaks to the illuminant shade of the information picture and has a similar measurement to the first RGB picture. The qualities at every pixel indicate the relating evaluated illuminant shading at this position. All in all, the illuminant hues in an area ought to be close because of the illuminant consistency. Be that as it may, picture recoloring can't keep up the illuminant consistency since the progressions of pixels are not indistinguishable.[17]

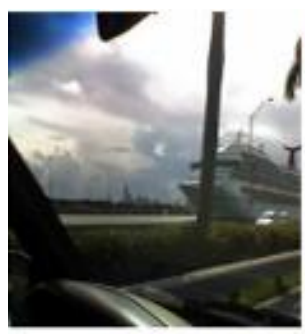

(a) Natural image

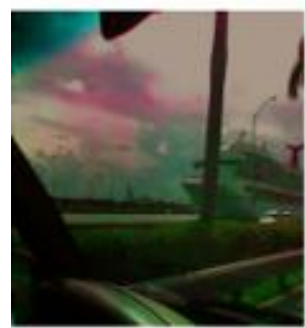

(c) Recolored image

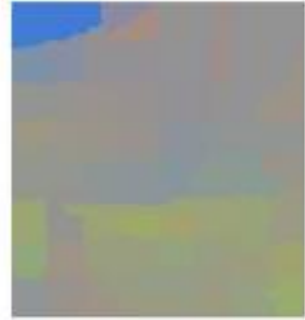

(b) IM of (a)

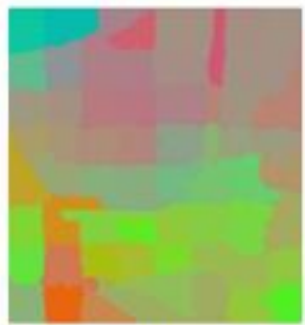

(d) IM of (c)
Fig 3.3 Comparision of IM's

\subsection{DEEP CNN MODEL}

A straightforward extendable library for preparing and assessing Deep Convolutional Neural Networks focussing on picture order and identification, RecDeNet is fit for supplanting picture portrayal methods.

In the proposed framework existing imitation recognition strategies will embrace hardly any desciption systems to aggregate the information accomplished by verification estimators an additionally every single depiction procedure has its own confinements and disadvantages. CNNS have indicated ubiquity in picture grouping and the other PC vision undertakings. Prior neural systems utilizes the genuine pic in RGB channels as the contribution as it is comprising of the data about picture as shading and compositional highlights.

For a picture to be judged, the distinction pictures (DIs) and the illuminant map (IM) are determined right off the bat.[18]

\section{EXPERIMENTAL RESULTS}

In this project we consider recoloured image dataset, which contains a combination of colored and recoloured image sets.

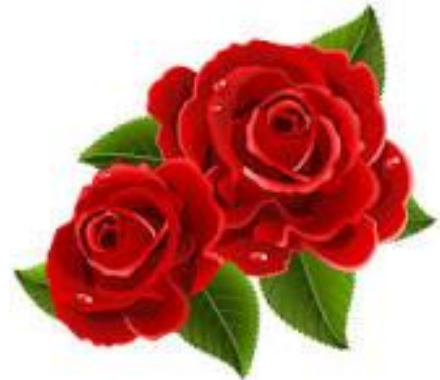

Fig 4.1 Input Image

Feature Extraction is done here. The input image is resized to $142 \times 147$ and changed from RGB to BGR.

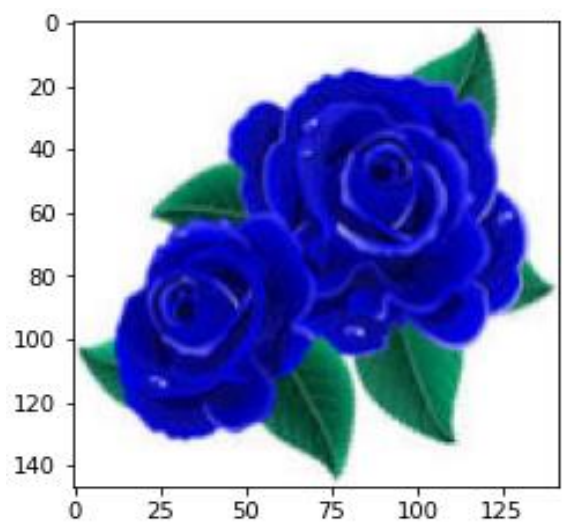

Fig 4.2 Resized Image

Interchannel Correlation is applied here. And DI and IM of the resized image is obtained.

The DI image is converted from BGR to Gray. 


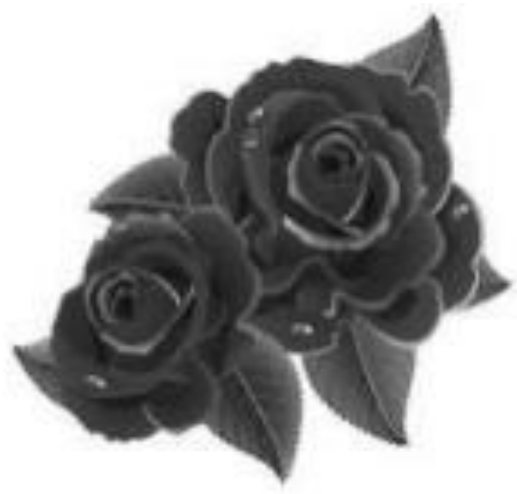

Fig 4.3 Gray Image

The similarity index is checked and it should be 1.0. From the resized original image IM is generated, i.e. our illuminant image.

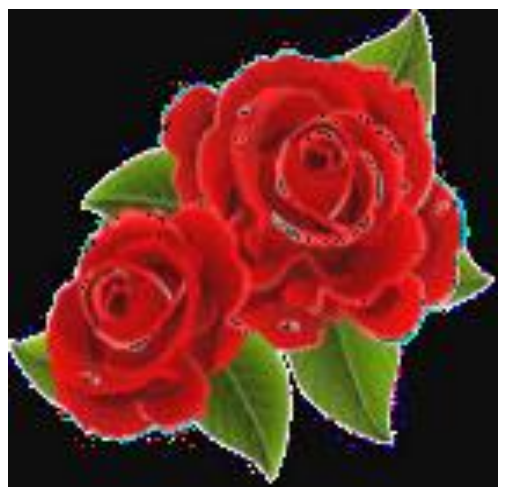

Fig 4.4 Illuminant Image

The fusion of all the images is done using their coefficients. The fused image is obtained.

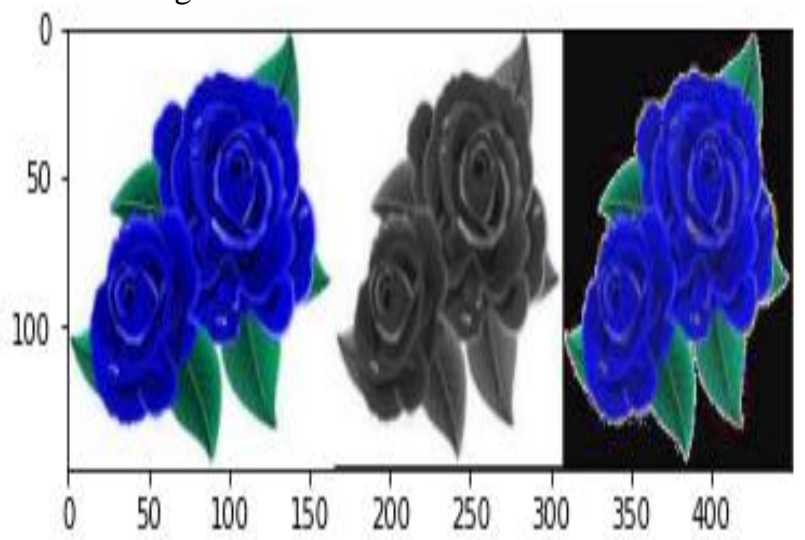

Fig 4.5 Fusion Image
The fused image is converted from BGR to RGB

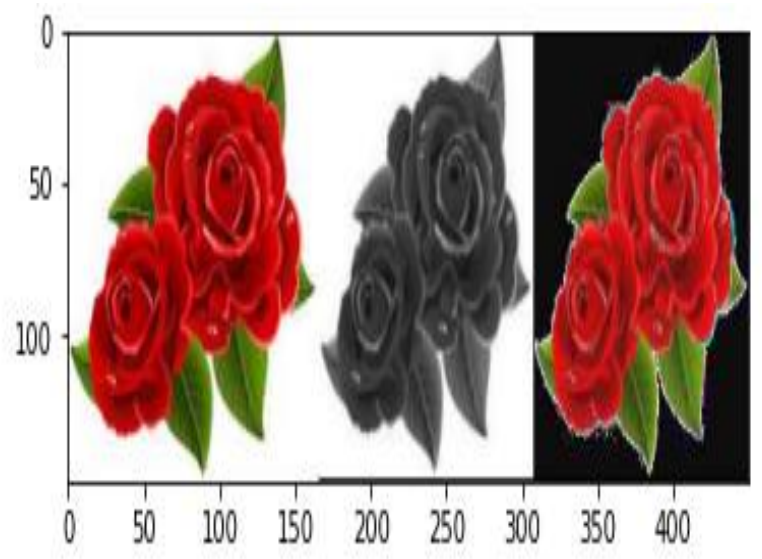

Fig 4.6 RGB Image

The RGB output for image is converted to a HSV plot.

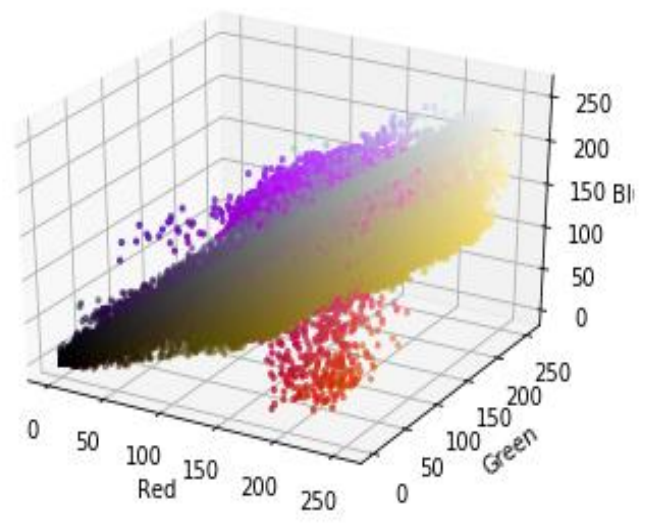

Fig 4.7 HSV Plot

\section{CONCLUSION}

Here we have introduced a strategy called as novel profound learning method for the recolored picture recognition. Here we utilize two systems which are called as between channel relationship and enlightenment consistency are gathered to help include extraction and furthermore we expand the structure guideline of our recolored identification organize and progressively confirm the proportion by directing different number of investigations and in further, the two shaded datasets with the various assets were produced and the high productivity of our recolored discovery arrange clarifies the adequacy and improvement of our model. Here we trust our straightforward and successful recolored discovery system will helps as a strong standard and will bolster as future research in recolored picture identification. At long last our future work will focuses on building up a progressively viable 


\section{International Journal of Engineering Applied Sciences and Technology, 2020 Vol. 5, Issue 1, ISSN No. 2455-2143, Pages 462-466 \\ Published Online May 2020 in IJEAST (http://www.ijeast.com)}

system engineering and furthermore looking for elevated level signals for better outcomes.

\section{ACKNOWLEDGEMENT}

Authors gratefully acknowledge the computational facility created in the college under DST's FIST Programme (SR/FST/College-2017/28(C)) which helped them to carry out the work. Authors thank the management of IARE for their support and kind encouragement.

\section{REFERENCES}

[1] E. Reinhard, M. Ashikhmin, B. Gooch, and P. Shirley, (2001) "Color transfer between images," IEEE Computer Graphics Applications, vol. 21, no. 5, pp. 34-41.

[2] F. Pitie, A. C. Kokaram, and R. Dahyot, (2007) "Automated colour grading using colour distribution transfer," Comput Vis Image Underst, pp. 123-137.

[3] M. P. Rao, A. N. Rajagopalan, and G. Seetharaman, (2014) "Harnessing motion blur to unveil splicing," IEEE Transactions on Information Forensics and Security, vol. 9, no. 4, pp. 583-595.

[4] G. Muhammad, M. Hussain, and G. Bebis, (2012) "Passive copy move image forgery detection using undecimated dyadic wavelet transform," Digital Investigation, vol. 9, no. 1, pp. 49-57.

[5] G. Cao, Y. Zhao, R. Ni, and X. Li, (2014) "Contrast enhancement-based forensics in digital images," IEEE Transactions on Information Forensics and Security, vol. 9, no. 3, pp. 515-525.

[6] K. Simonyan and A. Zisserman,(2014) "Very deep convolutional networks for large-scale image recognition," Computer Science.

[7] X. Zhao, J. Li, S. Li, and S. Wang, (2010) "Detecting digital image splicing in chroma spaces," in Digital Watermarking - International Workshop, pp. 12-22.

[8] M. C. Stamm and K. J. R. Liu, (2010) "Forensic detection of image manipulation using statistical intrinsic fingerprints," IEEE Transactions on Information Forensics and Security, vol. 5, no. 3, pp. 492-506.

[9] J. S. Ho, O. C. Au, J. Zhou, and Y. Guo, (2010) "Interchannel demosaicking traces for digital image forensics," in IEEE International Conference on Multimedia and Expo, pp. 1475-1480.

[10] F. Pitie and A. C. Kokaram, (2007) "The linear mongekantorovitch linear colour mapping for example-based colour transfer," IETCVMP, pp. 1-9.

[11] X. An and F.Pellacini, (2008) "Appprop: all-pairs appearance-space edit propagation," ACM Transactions on Graphics, vol. 27, no. 3, pp. 15-19.
[12] R. Chamlawi, A. Khan, and I. Usman, (2010) "Authentication and recovery of images using multiple watermarks," Computers and Electrical Engineering, vol. 36, no. 3, pp. 578-584.

[13] G. S. Spagnolo and M. D. Santis, (2011) "Holographic watermarking for authentication of cut images," Optics and Lasers in Engineering, vol. 49, no. 12, pp. 1447-1455.

[14] L. Rosales-Roldan, M. Cedillo-Hernandez, M. NakanoMiyatake, H. Perez-Meana, and B. Kurkoski, (2013) "Watermarking-based image authentication with recovery capability using halftoning technique," Signal Processing Image Communication, vol. 28, no. 1, p. 6983.

[15] C. S. Lu and H. Y. M. Liao, (2013) "Structural digital signature for image authentication: an incidental distortion resistant scheme," IEEE Transactions on Multimedia, vol. 5, no. 2, pp. 161-173.

[16] X. Wang, J. Xue, Z. Zheng, Z. Liu, and N. Li, (2012) "Image forensic signature for content authenticity analysis," Journal of Visual Communication and Image Representation, vol. 23, no. 5, pp. 782-797.

[17] M. Sengupta and J. Mandal, (2013) "Authentication through hough transformation generated signature on g-let d3 domain," Procedia Technology, vol. 10, pp. 121-130.

[18] Z. P. Zhou and X. X. Zhang, (2010) "Image splicing detection based on image quality and analysis of variance," in International Conference on Education Technology and Computer, pp. 242-246.

[19] C.-M. Pun, X.-C. Yuan, and X.-L. Bi, (2015) "Image forgery detection using adaptive over segmentation and feature point matching," IEEE Transactions on Information Forensics and Security, vol. 10, no. 8, pp. 1705-1716.

[20] Y. Cao, T. Gao, L. Fan, and Q. Yang, (2012) “A robust detection algorithm for copy-move forgery in digital images," Forensic science international, vol. 214, no. 1, pp. 33-43. 